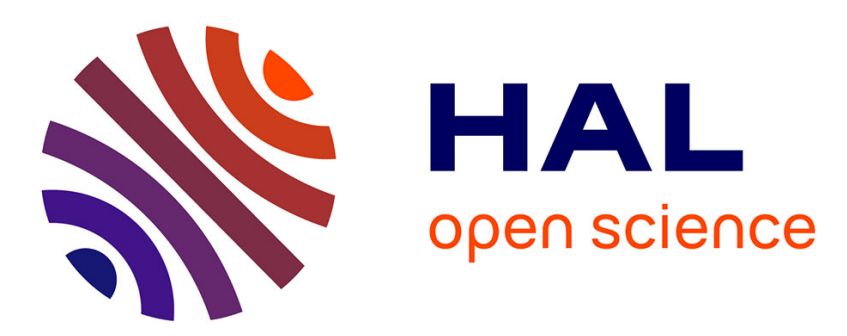

\title{
Single-shot CEP drift measurement at arbitrary repetition rate based on dispersive Fourier transform
}

Máté Kurucz, Szabolcs Tóth, Roland Flender, Ludovít Haizer, Balint Kiss, Benjamin Persielle, Eric Cormier

\section{- To cite this version:}

Máté Kurucz, Szabolcs Tóth, Roland Flender, Ludovít Haizer, Balint Kiss, et al.. Single-shot CEP drift measurement at arbitrary repetition rate based on dispersive Fourier transform. Optics Express, 2019, 27 (9), pp.13387-13399. 10.1364/OE.27.013387 . hal-02271070

\section{HAL Id: hal-02271070 \\ https://hal.science/hal-02271070}

Submitted on 26 Aug 2019

HAL is a multi-disciplinary open access archive for the deposit and dissemination of scientific research documents, whether they are published or not. The documents may come from teaching and research institutions in France or abroad, or from public or private research centers.
L'archive ouverte pluridisciplinaire HAL, est destinée au dépôt et à la diffusion de documents scientifiques de niveau recherche, publiés ou non, émanant des établissements d'enseignement et de recherche français ou étrangers, des laboratoires publics ou privés. 


\title{
Single-shot CEP drift measurement at arbitrary repetition rate based on dispersive Fourier transform
}

\author{
MÁté KuRucz, ${ }^{1}$ SzABolcs Tóth, ${ }^{1}$ Roland Flender, ${ }^{1}$ LUdovít HAIZER, ${ }^{1}$ \\ BÁlint Kiss, ${ }^{1}$ Benjamin Persielle ${ }^{2}$ And Eric Cormier ${ }^{2, *}$ \\ ${ }^{1}$ ELI-ALPS, Dugonics tér 13, 6720 Szeged, Hungary \\ ${ }^{2}$ CELIA, Université de Bordeaux - CNRS - CEA, 33405 Talence, France \\ *Eric.Cormier@u-bordeaux.fr
}

\begin{abstract}
This paper presents a single-shot technique for measuring CEP. The Temporal dispersion based One-shot Ultrafast Carrier envelope phase Analysis method (TOUCAN) is an arbitrary repetition rate single-shot CEP drift measurement technique based on dispersive Fourier transformations and has been experimentally tested at $100 \mathrm{kHz}$. TOUCAN was validated by a direct comparison of decimated data with an independent traditional CEP drift measurement technique. The impact of a temporal jitter on the CEP drift measurement is investigated and a new mitigation technique is shown to produce high accuracy jitter-free CEP drift extraction.
\end{abstract}

(C) 2019 Optical Society of America under the terms of the OSA Open Access Publishing Agreement

\section{Introduction}

High-field physics experiments e.g., attoscience, often require amplified few cycle laser pulses at the millijoule energy level [1]. The outcome of these experiments can drastically be altered by a change in the field amplitude and also by drifts or fluctuations of the carrier envelope phase (CEP) [1,2]. As a consequence, on top of the CEP stabilization scheme (passive or active), parallel single-shot CEP measurement and pulse tagging might become necessary.

One of the most widespread CEP drift measurement method is based on f-to-2f selfreferential interferometry [3], where the CEP slip is measured by a heterodyne detection scheme. The single-shot version of a f-to-2f interferometer extracts the phase information by optical spectroscopic method [4,5] i.e. using angular dispersion to map the spectrum onto a spatial dimension. The detection of the spatially dispersed signal usually relies on a detector array, e.g., a charge-coupled device (CCD). The measurement rate of such device is limited by the delay between measurements, which is usually on the hundred microseconds scale. Single-shot CEP drift measurement and pulse tagging above $10 \mathrm{kHz}$ is very challenging to achieve with current technology. Even when replacing the detector array with two photomultipliers, which in principle mitigates this limitation, the demonstrated fastest singleshot detection was at $10 \mathrm{kHz}[6]$.

In the last decade, Stereo-ATI, a new CEP detection technique has been developed, which encodes the phase in an electron spectrum of the above-threshold ionization (ATI) of a noble gas [7-9]. This method allows real time single-shot measurements of the CEP at high repetition rate and is independent of pulse energy to phase coupling inherent to f-to-2f type interferometric measurements. This coupling leads to spurious CEP noise measurement for a system which uses supercontinuum generation for spectral broadening [10]. Single-shot real time stereo-ATI CEP measurements on a $100 \mathrm{kHz}$ repetition rate system have recently been demonstrated and it is claimed, based on the measurement time, that theoretical limit of the acquisition rate is $10 \mathrm{MHz}$ [11]. However, this technique requires two time of flights tubes to be connected to the interaction chamber, under vacuum, resulting in a large expansive device. 
Also few cycle pulses with at least $30 \mu \mathrm{J}$ energy are required for CEP retrieval [9], which makes this diagnostic method unsuitable for a significant proportion of current laser systems.

A different approach for high repetition rate single-shot CEP measurement has been taken which is based on Dispersive Fourier Transform (DFT) [12-15]. The temporal dispersion based one-shot ultrafast carrier envelope phase analysis method (TOUCAN) allows singleshot CEP drift extraction up to gigahertz repetition rates from f-to-2f spectral interference. The concept is demonstrated by recording the CEP drift of 4 cycles pulses at $3.2 \mu \mathrm{m}$ from an OPCPA laser operating at $100 \mathrm{kHz}$.

\section{Concept}

Linear optical interferometry cannot provide information about the CEP so a nonlinear optical effect has to be employed [3]. In f-to-2f self-referential interferometry, a fraction of the laser pulse is frequency doubled while another fraction is spectrally broadened until the blue part reaches the doubled frequency (i.e. broadened over one octave). The interference signal is created by overlapping these two pulses in the temporal, spatial, and spectral domain. The one dimensional temporal evolution of the fundamental laser pulse is given by

$$
E_{\omega}(t)=A(t) \cdot \exp \left(-i\left(\omega_{0} t+\varphi\right)\right)
$$

where $\mathrm{A}(\mathrm{t})$ is the temporal envelope of the pulse, $\omega_{0}$ is the central angular frequency and $\varphi$ stands for CEP. In the frequency domain the field reads:

$$
\mathrm{E}_{\omega}(\omega)=\mathrm{A}_{\omega}(\omega) \cdot \exp (-\mathrm{i} \varphi)
$$

Under perfect conditions, the second harmonic ( $\mathrm{SH}$ ) pulse is centered at twice the frequency of the original. The resultant phase is twice that of the fundamental pulse and also shifted by an offset. The complex spectrum of the second harmonic electric field is expressed as:

$$
\mathrm{E}_{2 \omega}(\omega)=\mathrm{A}_{2 \omega}(\omega) \cdot \exp (-\mathrm{i}(2 \varphi+\pi / 2))
$$

The above equation describes a process, where dispersion is negligible. In reality material dispersion introduces different constant phase shift for the fundamental and SH pulses. The spectra of these pulses may overlap if the spectrum is either sufficiently broad or if it is broadened by supercontinuum generation. The overlapping region will exhibit a spectral interference pattern, where the fringe period depends on the relative delay between the fundamental and the SH pulse. The resulting signal as detected by an integrating device is described by:

$$
\begin{aligned}
I_{\mathrm{f} 22 \mathrm{f}}(\omega) & =\left(\mathrm{E}_{\omega}(\omega)+\mathrm{E}_{2 \omega}(\omega)\right) \overline{\left(\mathrm{E}_{\omega}(\omega)+\mathrm{E}_{2 \omega}(\omega)\right)} \\
& =I_{\omega}(\omega)+\mathrm{I}_{2 \omega}(\omega)+2 \sqrt{\mathrm{I}_{\omega}(\omega) \cdot \mathrm{I}_{2 \omega}(\omega)} \cdot \cos \left(\varphi+\varphi_{\mathrm{D}}+\omega \cdot \Delta \mathrm{t}+\pi / 2\right)
\end{aligned}
$$

where the $\Delta \varphi_{\mathrm{D}}$ is the phase difference between pulses introduced by the material dispersion. The third term in this equation creates a spectral intensity modulation where the phase of this pattern is in fact linearly related to $\varphi$. Thus extracting the phase of the spectral modulation provides the CEP value of the original pulse shifted by an unknown constant value.

Conventional techniques would read the spectrum with an optical spectrometer with a limiting reading frequency of 1 or $10 \mathrm{kHz}$. The TOUCAN technique, which is based on dispersive Fourier transform, can reach higher data acquisition rate, because there is no dependence on an optical spectrometer. The DFT itself is performed by propagating the laser through a dispersive optical element with very high group delay dispersion (GDD). When the laser pulse enters a dispersive medium, different frequency components travel at different phase velocities, accumulating different time delays. Consequently, the pulse duration is stretched by orders of magnitude above its original value. The resulting temporal shape will only be the function of the original spectral shape and the dispersion. Dispersion can be 
considered as a transform, where each spectral component is mapped to the temporal domain. The relation between optical frequency and the corresponding time delay can be expressed in the far-field approximation [13] as:

$$
\begin{aligned}
\mathrm{T}(\omega)= & \operatorname{GD}\left(\omega_{0}\right)+\operatorname{GDD}\left(\omega_{0}\right) \cdot\left(\omega-\omega_{0}\right)+ \\
& +\frac{1}{2} \cdot \operatorname{TOD}\left(\omega_{0}\right) \cdot\left(\omega-\omega_{0}\right)^{2}+\frac{1}{6} \cdot \operatorname{FOD}\left(\omega_{0}\right) \cdot\left(\omega-\omega_{0}\right)^{3}+\ldots
\end{aligned}
$$

where $\omega$ is the angular frequency, $\omega_{0}$ is the central angular frequency, $\mathrm{T}$ is accumulated time delay of the laser pulse traveling through the medium. The phase derivatives present in the equation are abbreviated as GD: group delay (first order), GDD: group delay dispersion (second order), TOD third order dispersion, FOD fourth order dispersion, etc...The effect of the third and even higher order dispersion can be neglected, if its effect is marginal compared to the effect of the first order GD and the second order GDD. Thus, the frequency-time mapping becomes linear in frequency as:

$$
\mathrm{T}(\omega)=\mathrm{GD}\left(\omega_{0}\right)+\mathrm{GDD}\left(\omega_{0}\right) \cdot\left(\omega-\omega_{0}\right)
$$

Consequently, the spectral and temporal shape become congruent and by dispersing the output signal of an f-to- $2 \mathrm{f}$ interferometer, the spectral interference pattern is mapped to the temporal domain. The stretched pulse duration can reach hundreds of nanoseconds, which allows for reading the temporal modulation with a relatively slow photodetector. The produced electric signal can then be digitized by an analog-to-digital (A/D) converter with a bandwidth at least twice the modulation frequency. The CEP drift can be extracted from the phase of the digital waveform, provided the dispersion is fully characterized.

\section{Experimental setup}

The concept has been demonstrated on a high-repetition rate system producing CEP stable few-cycle pulses equipped with a conventional f-to- $2 \mathrm{f}$ measurement device for comparison and validation. The experimental setup comprises a commercial state-of-the-art mid-infrared (MIR) laser system operated at ELI-ALPS [16] and the MIR laser setup is shown in Fig. 1(a). The system produces passively CEP stabilized $150 \mu \mathrm{J}, 4$ optical-cycle (42 fs) pulses centered at $3.2 \mu \mathrm{m}$ at a repetition rate of $100 \mathrm{kHz}$. This OPCPA system uses a Dazzler (Fastlite) [17] to accurately shape the spectral amplitude and control the phase, including the CEP. Therefore, the CEP of the output pulses can be set to an arbitrary value in a very simple way. A sampled portion of the compressed output is sent to a commercial CEP measurement device based on a f-to-2f interferometer and a conventional grating spectrometer (Fringeezz from Fastlite) [18]. This device is able to record single shot CEP values at an under sampled repetition rate of 10 $\mathrm{kHz}$, that is one pulse out of 10 on the $100 \mathrm{kHz}$ laser system, and it is used for calibration and cross-checking purposes.

Another fraction of the laser output is steered towards the TOUCAN setup. The experimental arrangement of this single-shot CEP drift measurement device is shown in Fig. 1(b). The device consists of a f-to-2f interferometer and a DFT measuring system. Dispersion is achieved in the current case by propagation in a dispersion compensation fiber (DCF, FSCDCM-014D, OFS/Lucent). The spectral modulations originating from the f-to-2f interferometer (described in the concept section) are mapped into the temporal domain and detected by an InGaAs photodiode (DET01CFC, Thorlabs). The temporal waveform is then digitized and recorded with a $600 \mathrm{MHz}$ oscilloscope (RTO2004, R\&S). The oscilloscope was operated in segmented memory mode (ultra segmentation) in order to record every waveform at the full repetition rate of $100 \mathrm{kHz}$ [19]. 


\section{Optics EXPRESS}

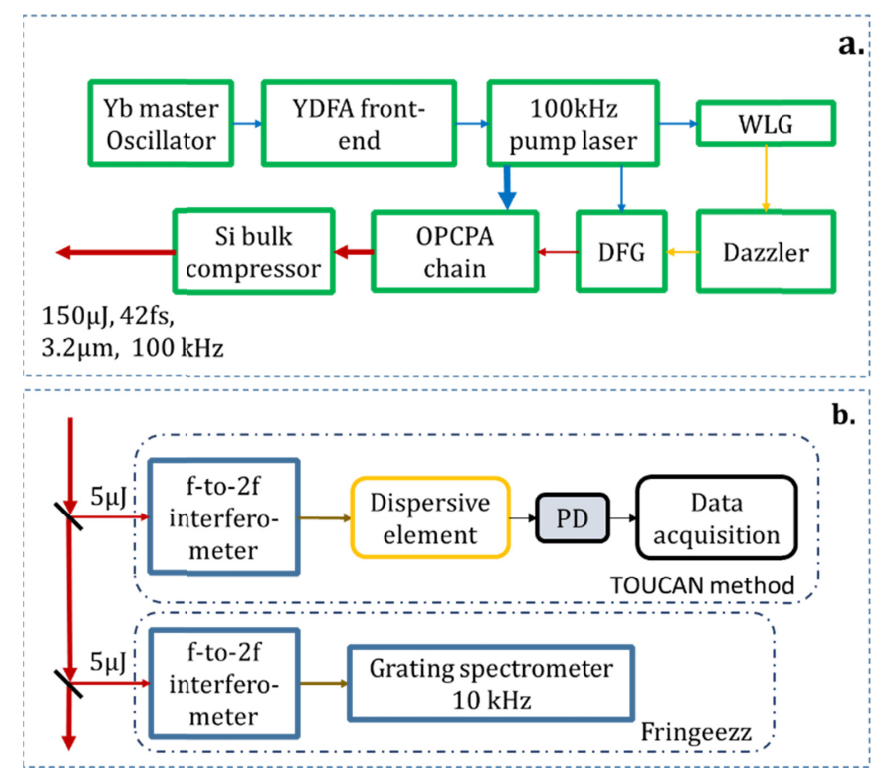

Fig. 1. (a) Scheme of the MIR laser system. (b) Schematics of the TOUCAN single shot CEP drift measurement and Fringeezz grating spectrometer based cross-checking.

A typical $\mathrm{f}$-to- $2 \mathrm{f}$ interference pattern recorded using the grating spectrometer is shown in Fig. 2. for different CEP values. The spacing of the interference pattern reveals that the time delay is 178 fs between the fundamental and second harmonic pulse. The pattern is linearly shifted with the CEP value as predicted by Eq. (4).

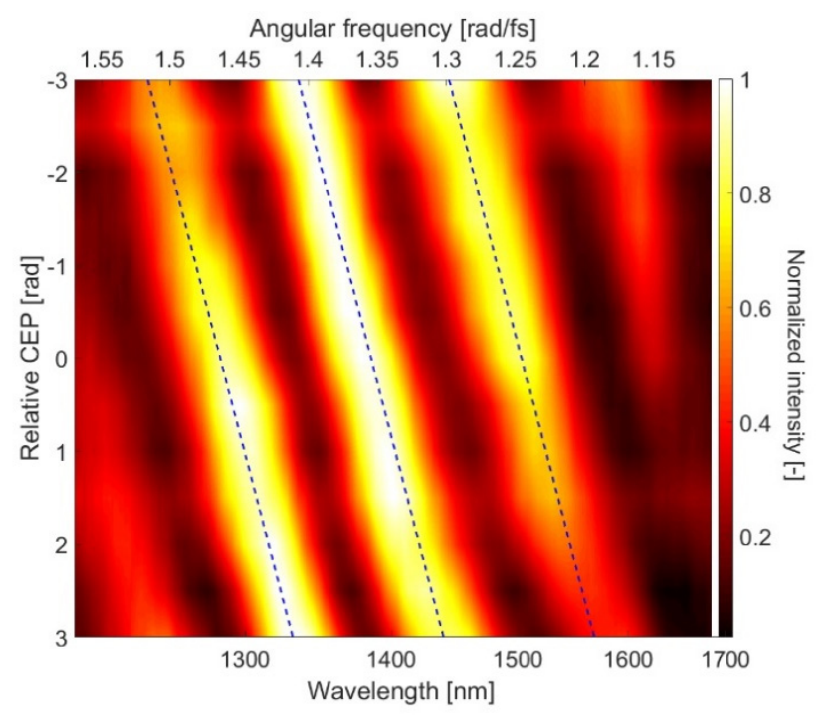

Fig. 2. f-to-2f spectral interference pattern recorded with grating spectrometer for different CEP values. The dashed lines are fitted on spectral fringe maxima. Linear correspondence between CEP and the position of these maxima can be observed.

\section{Dispersion characterization}

The dispersive element in the experimental DFT setup is a $1.88 \mathrm{~km}$ long DCF. The DFT technique requires an accurate characterization of the fiber dispersion to retrieve the spectral content from the temporal waveform. Several methods are commonly used to measure the 
dispersion based on interferometric techniques [20]. An ideal dispersion measurement technique would propagate a frequency comb in the fiber and detect on the oscilloscope the delays between each successive frequency line. Such a comb was not available, so the modulated spectrum from the f-to-2f, mimicking a periodic spectral etalon, was used.

The $\sim 100 \mathrm{~ns}$ temporal waveform was measured with the photodiode and oscilloscope. The spectral measurements were made using a high resolution optical spectrum analyzer (AQ6375 B, Yokogawa). Both measurements were low-pass filtered during processing to remove the high frequency noise. The modulation, as predicted by the preliminary calculation, is resolvable with the photodiode and the A/D converter of the oscilloscope. The fiber adds a positive chirp to the signal so the temporal waveform is reversed when compared to the spectrum, if plotted as a function of wavelength. Superposition is thus visualized with the time axis reversed (Fig. 3.). An excellent overlap is obtained when the frequency dependent time delay function (Eq. (5).) is fully retrieved.

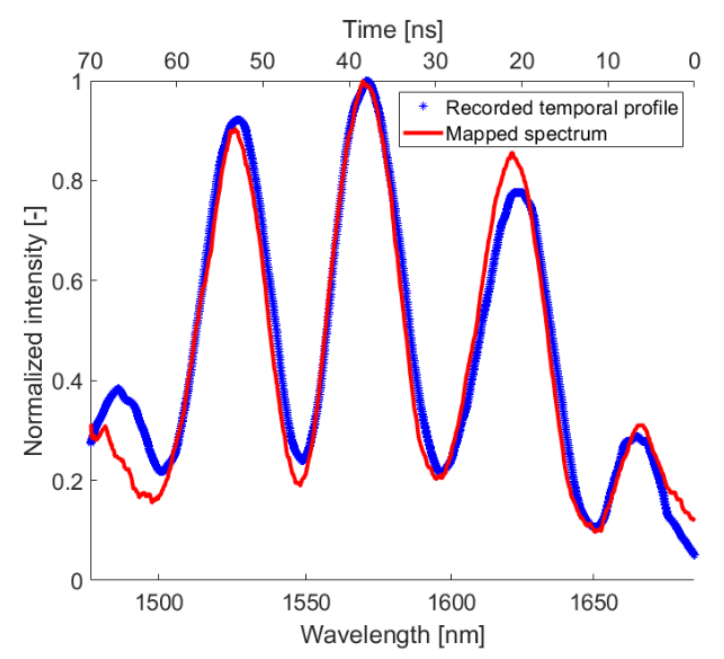

Fig. 3. f-2-2f interference pattern used to determine the dispersion of the fiber. Superimposed are the temporal waveform recorded with a photodiode and digitalized by the oscilloscope plotted with a reversed time axis and the spectrum recorded with an optical spectrum analyzer.

The different dispersion orders of the equation are determined by fitting the dispersed spectral signal over the whole temporal signal according to Eq. (5) using an iterative algorithm, shown in Fig. 4. In the first step, the dispersion parameters are given initial values and the resultant temporal profile is calculated. The difference between the measured and the calculated temporal signals are used as a fitting error, which is subsequently minimized via the iteration process. The most intense peaks have the highest weight in the fitting process, making local difference smaller for the main peaks when compared to the side peaks. The comparative measurement of the interference pattern was done for 13 data sets with different CEP values. 


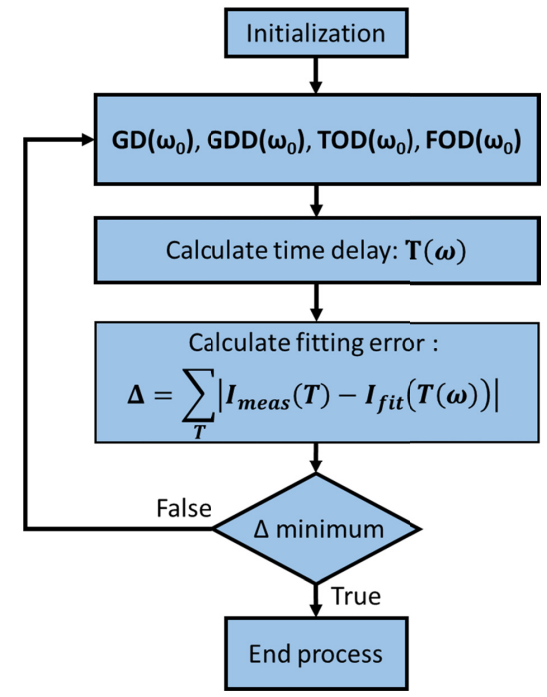

Fig. 4. Schematic representation of the iterative algorithm to determine the various dispersion orders.

The retrieved time delay function and dispersion parameter is plotted on Fig. 5. and includes dispersion coefficients up to the fourth order. The dispersion value, provided by the manufacturer, is $-340 \mathrm{ps} / \mathrm{nm}$ at the wavelength of $1550 \mathrm{~nm}$, which is very close to the -339.3 $\mathrm{ps} / \mathrm{nm}$ calculated from the fitting parameters. The different dispersion coefficient from the time delay measurement could also be extracted: a GDD of $432.7 \mathrm{ps}^{2}$, a TOD of $-0.537 \mathrm{ps}^{3}$ and a FOD of $-0.0084 \mathrm{ps}^{4}$. The contribution of the FOD and the higher order dispersion coefficients are marginal to the time delay and as a consequence, Eq. (6) proved to be insufficient to characterize the dispersion of the fiber so Eq. (5) was used up to the TOD contribution.

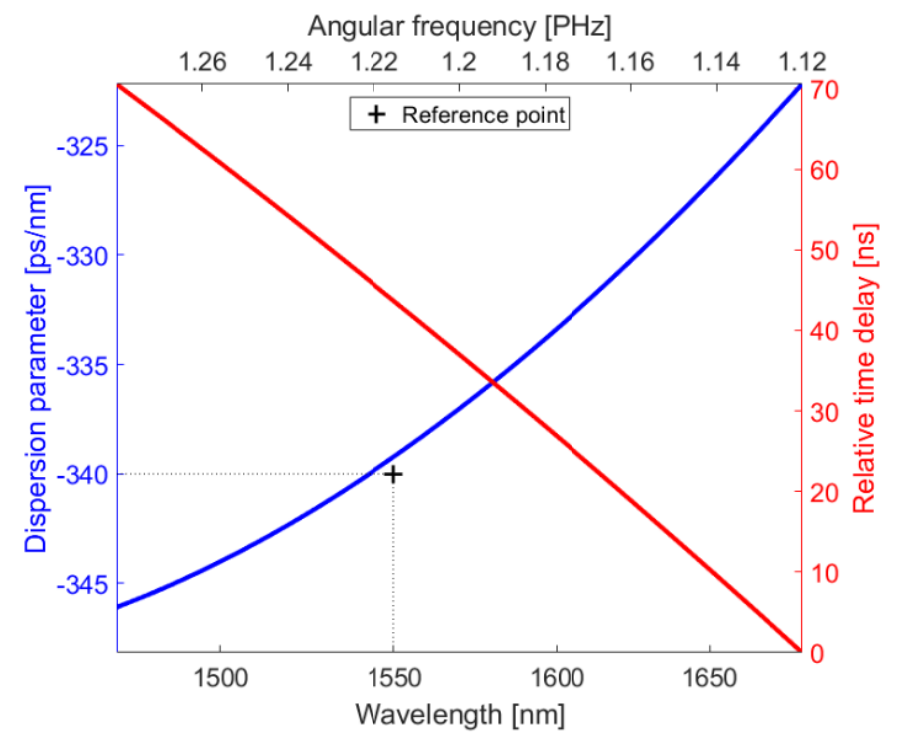

Fig. 5. Retrieved time delay as the function of the angular frequency (red) and the corresponding dispersion parameter (blue) for the FSC-DCM-014D fiber. The black cross is the dispersion parameter provided by the manufacturer. 


\section{CEP drift measurement}

The CEP drift of the pulses is measured with a single shot at the same time using TOUCAN at the full repetition rate of $100 \mathrm{kHz}$ and with the Fringeezz device at $10 \mathrm{kHz}$ sampling rate. The two data sets, gathered by the two independent devices, contains the CEP drift of the pulses for a $1 \mathrm{~s}$ time period, which was ultimately limited by the oscilloscope internal memory. However, one second of data corresponds to 100,000 shots which is ample for statistical analysis of the short term CEP change. The synchronization of the two CEP data sets was achieved by creating a few millisecond long event where the seed for the OPA stages are cut by the LN-AOPDF (Dazzler, Fig. 1(a).). This "no-seed" event was distinguishable in post-processing and facilitated synchronization between data sets.

In order to correlate the results from systems with different repetition rates, the $100 \mathrm{kHz}$ TOUCAN CEP data sets was decimated [21], as one of the 10 possible measurements coincides with the Fringeezz data. This approach produces the highest correlation between data sets, acquired by using circular correlation formula [21] due to the circular algebraic properties of CEP. The formula for calculating circular correlation coefficient is shown in Eq. (7).

$$
R_{\text {cor }}=\frac{\sum_{i=1}^{n} \sin \left(\alpha_{i}-A\right) \cdot \sin \left(\beta_{i}-B\right)}{\sqrt{\sum_{i=1}^{n} \sin ^{2}\left(\alpha_{i}-A\right) \cdot \sum_{i=1}^{n} \sin ^{2}\left(\beta_{i}-B\right)}}
$$

$\alpha_{\mathrm{i}}$ and $\beta_{\mathrm{i}}$ are both sets of samples, with circular characteristics, while A and B are circular means [22] of these samples respectively. The $\mathrm{R}_{\text {cor. }}$ circular correlation coefficient value can vary between -1 (anticorrealtion) and 1 (correlation), and if the deviation from the circular mean is small, the resulting value is close to unwrapped Pearson correlation coefficient.

Figure 6. shows the correlation coefficient of the ten possible decimated data sets. Out of the ten possible decimated sets, the second highest correlation coefficient reaches only 0.491 , compared to the 0.982 at the maximum. This huge peak appeared in every experiment consistently, allowing the selection of the exact corresponding data sets reliably. From this point on, the down sampled data set with the highest correlation coefficient will be referred to as the decimated data set.

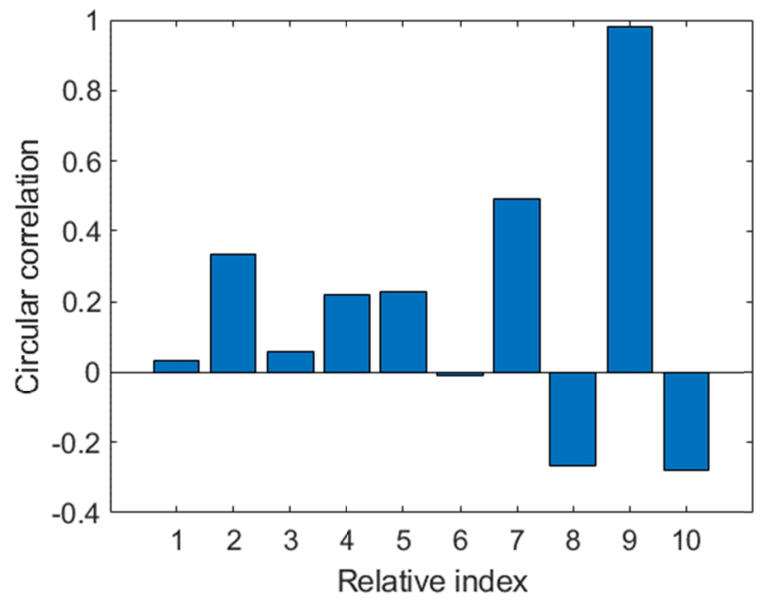

Fig. 6. Circular correlation coefficients between the Fringeezz CEP drift data set and the ten possible decimated $(10 \mathrm{kHz})$ CEP data set measured by the TOUCAN method. In this case, data set 9 matches the decimated Fringeezz CEP data.

The CEP fluctuation results are summarized in Table 1. The CEP noise is calculated from the $100 \mathrm{kHz}$ TOUCAN data set as well as the decimated data set but no significant difference 
is found as decimating a data set is not equivalent to low-pass filtering the data [23]. After decimation, high-frequency noise contributions are aliased into the lower frequency region, which means that the overall noise level does not change significantly. The measurements with the conventional technique gives a smaller CEP noise standard deviation than the TOUCAN method. The reported uncertainty of the Fringeezz measurement originate from the spectral resolution of the grating spectrometer, provided by the manufacturer. The uncertainty of the TOUCAN method originates from the temporal resolution of the recorded waveforms and partly on time jitter affecting the measurement. The measurement resolution is set by the time resolution of the oscilloscope. The frequency to time mapping is not perfectly linear so the CEP resolution differs at different time in the waveform. For instance, the CEP resolution ranges from 33 to $43 \mathrm{mrad}$ along the recorded waveform and is $38 \mathrm{mrad}$ at the center where the CEP value is extracted. Multiple data points are used for the CEP extraction so this value should only be considered as an upper bound for the uncertainty. The standard deviation of the time jitter is $126 \mathrm{ps}$, which translates to an additional $48 \mathrm{mrad}$ CEP noise. The combined random error can be calculated by taking the root sum of squares of these two uncertainties, which is also displayed in Table 1.

\begin{tabular}{lll}
\multicolumn{3}{c}{ Table 1. Measured CEP noise by different methods } \\
Fringeezz & $\begin{array}{l}\text { TOUCAN } \\
\text { decimated }\end{array}$ & $\begin{array}{l}\text { TOUCAN } 100 \\
\mathrm{kHz}\end{array}$ \\
\hline & & \\
$341 \pm 5 \mathrm{mrad}$ & $351 \pm 52 \mathrm{mrad}$ & $350 \pm 52 \mathrm{mrad}$ \\
\hline
\end{tabular}

The validation of TOUCAN with the Fringeezz measurements is not straightforward as data set is being compared with another undersampled data set. The CEP standard deviation calculated on both data sets can be identical although the data may be uncorrelated i.e., the measured Fringeezz $10 \mathrm{kHz}$ data set is shifted in time by several pulses compared to the decimated set. In this case, a correlation evaluation should identify the corresponding $10 \mathrm{kHz}$ data sets. However, the correlation coefficient is insufficient to warrant the correct calibration of the device because multiplying one of the data set by an arbitrary small number would still produce almost the same correlation value. Then, in order to certify the CEP calibration, a linear fitting was performed on the scatter plot of the corresponding data sets (Fig. 7.). The slope indicates the relative magnification of the measured CEP between the two measurement techniques. A slope of 1 would be a high correlation (all points scattered along a narrow line) would confirm a proper calibration, which was the case in almost all experiments. 


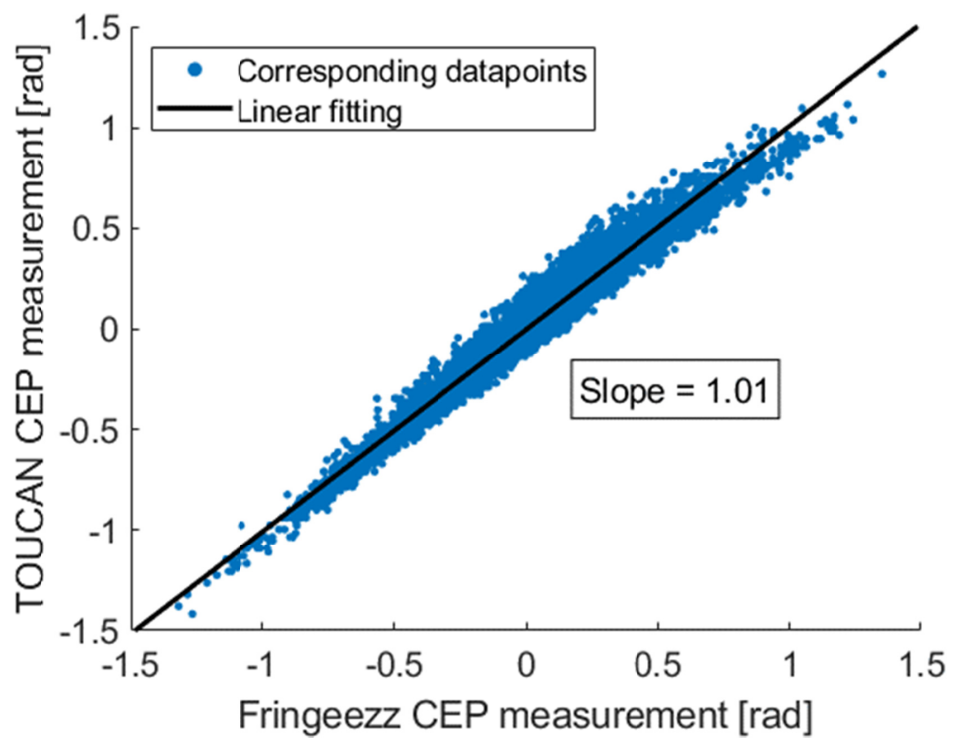

Fig. 7. The correlation of CEP data-points measured by the undersampled Fringeezz device and by TOUCAN technique. Linear fitting with slope of 1.01 is displayed with continuous black line.

The recorded CEP noise was also analyzed with the power spectral density (PSD) and the integrated phase noise (IPN) for the TOUCAN CEP measurement at $100 \mathrm{kHz}$ (Fig. 8(a).), and for the decimated TOUCAN and the Fringeezz measurement at $10 \mathrm{kHz}$ (Fig. 8(b).). Both PSD (on Fig. 8(b).) are almost completely overlapping over the entire range of data. Additionally, both methods exhibit large peaks at the same frequencies, further validating these results. However, these frequency components are distributed differently on the $100 \mathrm{kHz}$ PSD. This is attributed to aliasing of the actual noise components recorded above $5 \mathrm{kHz}$ (Nyquist frequency for the $10 \mathrm{kHz}$ measurement) and visible on the $100 \mathrm{kHz}$ PSD [23].

Noise contributions mostly stem from the resonance below $10 \mathrm{~Hz}$ (slow drift) and above 5 $\mathrm{kHz}$. One big motivation for real time single-shot CEP drift measurement is creating a feedback signal for CEP stabilization loop. Any such stabilization loop can cancel noise up to the Nyquist frequency. The integrated phase noise calculated from TOUCAN measurement reaches $88 \%$ of the full integral between $5 \mathrm{kHz}$ and $50 \mathrm{kHz}$. This spectral range of the phase noise could only be compensated with a $100 \mathrm{kHz}$ feedback-loop based on $100 \mathrm{kHz}$ single-shot CEP detection. 


\section{Optics EXPRESS}
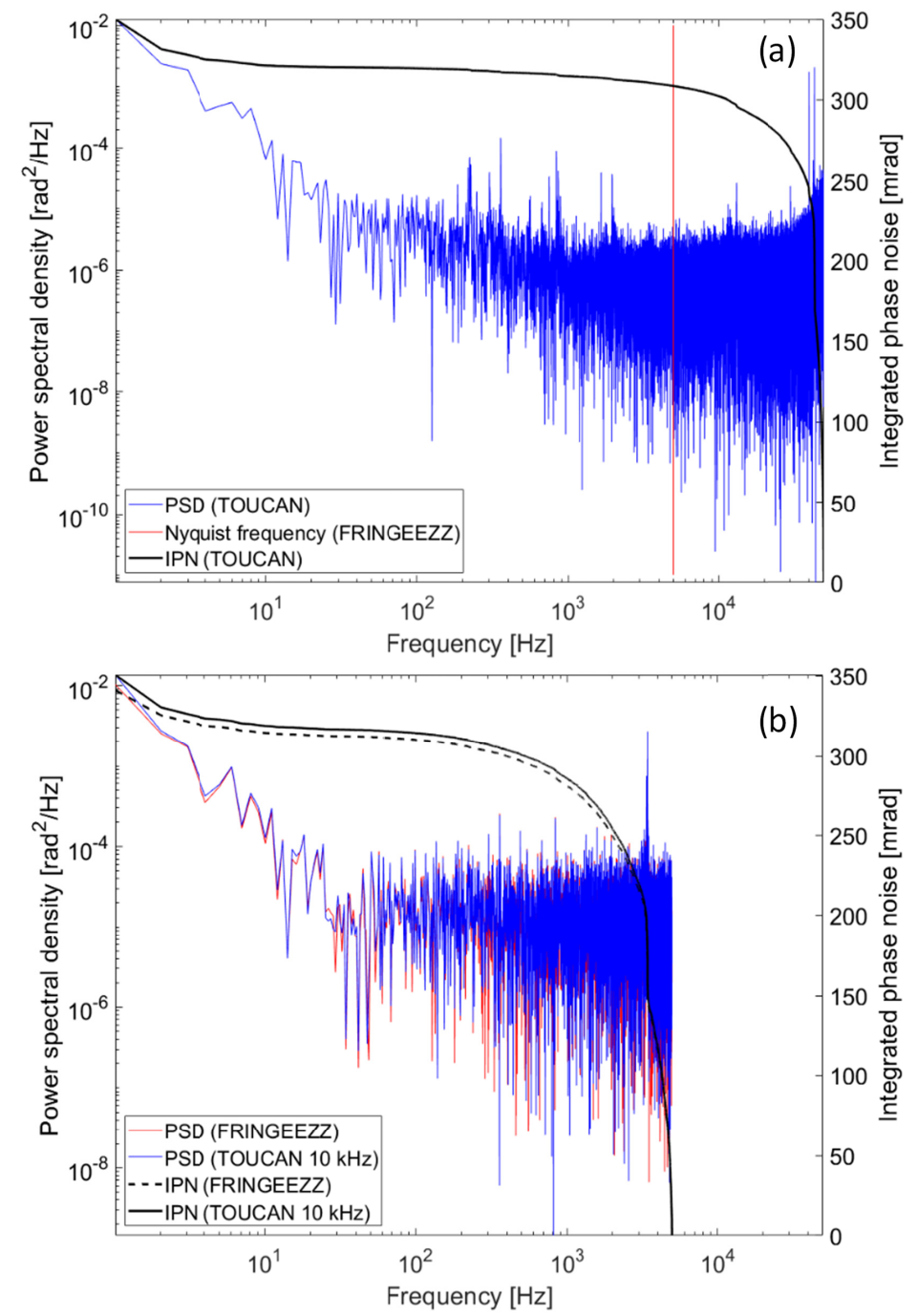

Fig. 8. (a) PSD of the CEP measured with the TOUCAN method at a $100 \mathrm{kHz}$ (blue line), and the corresponding IPN (black line). The Nyquist frequency of the Fringeezz is also displayed (red line) (b) PSD of the CEP measured with the TOUCAN method and decimated to $10 \mathrm{kHz}$ (blue line), and the corresponding IPN (black line). PSD measured with Fringeezz at $10 \mathrm{kHz}$ (red line), and the corresponding IPN (dashed black line).

\section{Time jitter compensation}

The timing system of the MIR laser provides $100 \mathrm{kHz}$ TTL signal (triggered from the master oscillator of the laser system) which was used to trigger the oscilloscope in the experiment. Unfortunately, any time jitter present between the output laser pulse and the TTL signal directly affects the position of the peaks and consequently the measured CEP drift. This can cause additional artificial CEP noise compared to a measurement using a conventional spectrometer. In order to evaluate and mitigate the impact of the time jitter a Fabry-Pérot etalon (uncoated $100 \mu \mathrm{m}$ thick YAG plate) is introduced before the DFT fiber to create a replica of the pulses with a known and fixed delay. The time delay between the two transmitted pulses generates a spectral interference, similar to the f-to-2f signal, although at a higher frequency. This superimposed modulation pattern is also transformed to the temporal 
domain in the DFT fiber, as shown on Fig. 9(a). The change in the phase of the high frequency modulation is only dependent on time jitter and completely independent on the change of the carrier envelope phase. In post-processing the two different frequency modulations are separated by Fourier spectral analysis. The original single modulation $f$-to- $2 \mathrm{f}$ signal is determined by employing a low-pass filtering, while single-shot time jitter was extracted from the high-pass filtered spectrum. Time domain statistical analysis can be performed by applying inverse Fourier transform on these separated spectra.

The shot-to-shot temporal displacement caused by time jitter can be used to shift the time domain of the filtered f-to- $2 \mathrm{f}$ signal. This way a truly jitter free single-shot CEP drift measurement can be obtained, mitigating one limitation of the TOUCAN approach. Compared to the uncorrected values, the time jitter corrected CEP drift data has lower uncertainty. Circular correlation coefficient is 0.99 , and the slope of linear fitting is 0.99 . Resulting statistical values are displayed in Table 2. Despite the improved statistics, the phase noise caused by the time jitter (Fig. 9(b).) is mostly overshadowed by the phase noise from other sources in the frequency domain. For this very reason the PSD and IPN of the jitter free measurements are mostly identical to the uncorrected CEP drift measurements.
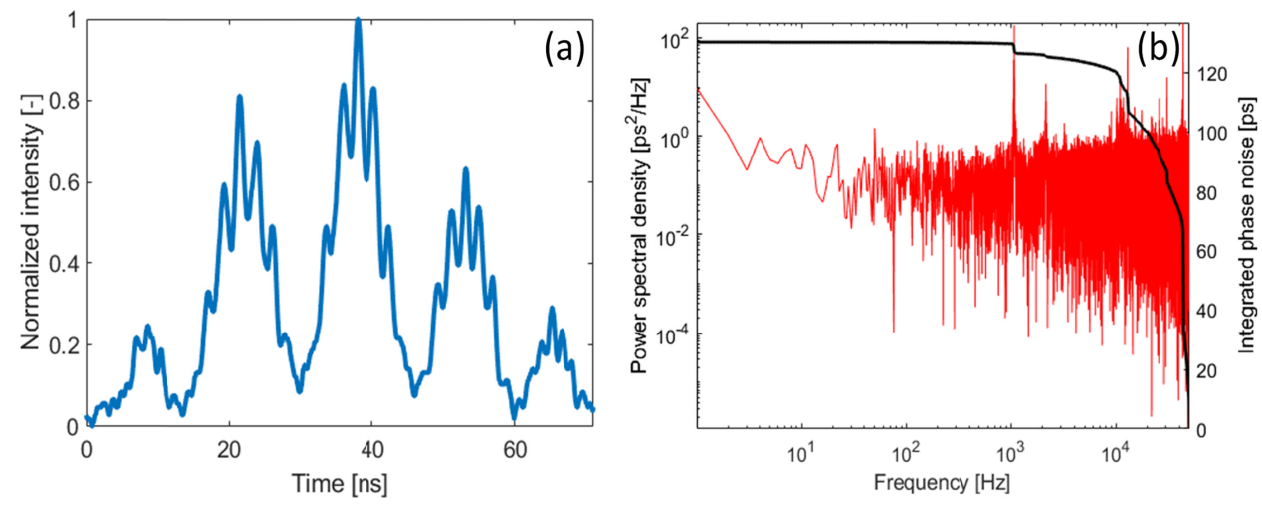

Fig. 9. (a) Double interference pattern with the CEP encoded in low frequency modulation, while the time jitter is encoded in the high frequency modulation. (b) Power spectral density and integrated phase noise function of time jitter.

Table 2. Time jitter compensated CEP noise measurement

\begin{tabular}{lll} 
Fringeezz & $\begin{array}{l}\text { TOUCAN } \\
\text { decimated }\end{array}$ & $\begin{array}{l}\text { TOUCAN } 100 \\
\mathrm{kHz}\end{array}$ \\
\hline $341 \pm 5 \mathrm{mrad}$ & $340 \pm 19 \mathrm{mrad}$ & $338 \pm 19 \mathrm{mrad}$ \\
\hline
\end{tabular}

The uncertainty of the TOUCAN measurement can be further improved to match or even exceed that of the Fringeezz device. The resolution of the recorded waveforms was limited by the bandwidth of the oscilloscope. The dispersion and consequently, the pulse duration can be increased by using a longer fiber, reducing the uncertainty of the measurement. Another approach is to use a data acquisition system specifically designed for continuous high resolution data acquisition and processing.

\section{Conclusions}

A new cost effective and simple method for CEP drift detection has been developed and verified. TOUCAN is capable of single-shot measurements at arbitrary repetition rate and was demonstrated on a state of the art CEP stable few-cycles laser system operating at $100 \mathrm{kHz}$ and emitting at $3.2 \mu \mathrm{m}$. The CEP extraction is done in post processing, so no real time CEP drift measurement and CEP stabilization can be performed at the moment. However, we are 
currently working on dedicated electronics able to extract the CEP on the fly at the full repetition rate.

The TOUCAN experimental setup designed for this $100 \mathrm{kHz}$ experiment is suitable without modifications for measurements at a repetition rate up to $10 \mathrm{MHz}$. The method can be possibly applied at even higher repetition rates with less dispersive fibers and faster scopes, with limitations imposed by the photodiode response time; the bandwidth of the dedicated DAQ hardware and analog data acquisition and recording times. For this reason, the prospect of $\mathrm{MHz}$ or even $\mathrm{GHz}$ single-shot CEP drift detection with the TOUCAN technique warrants further investigations. Also, many other laser systems produce few cycle pulses directly from an OPCPA or via postcompression however in the near IR spectral range $(800 \mathrm{~nm}$ to 1030 $\mathrm{nm}$ ) with some of them operated at repetition rates in excess of $100 \mathrm{kHz}$. Further investigations are being carried out to transfer this concept to the single shot measurement of the CEP noise to this spectral range. In fact, many CEP sensitive experiments may require an actual pulse tagging if the laser system CEP noise cannot be reduced sufficiently.

The energy needed for the CEP detection with the TOUCAN method was $2.5 \mu \mathrm{J}$ minimum with the current setup. This can be significantly lowered as f-to- $2 \mathrm{f}$ interferometers with specialized optics require only $\mathrm{nJ}$ energies, and avalanche photodetectors sensitive at the telecom wavelength are readily available. With further development the energy requirements can be decreased orders of magnitude below that of the single-shot stereo-ATI phase meter.

\section{Funding}

European Union - ELI-ALPS project (GOP-1.1.1.-12/B-2012- 0001, GINOP-2.3.6-15-201500001), European Regional Development Fund, Laserlab-Europe EU-H2020 654148, Institut Universitaire de France.

\section{References}

1. A. Baltuška, T. Udem, M. Uiberacker, M. Hentschel, E. Goulielmakis, Ch. Gohle, R. Holzwarth, V. S. Yakovlev, A. Scrinzi, T. W. Hänsch, and F. Krausz, "Attosecond control of electronic processes by intense light fields," Nature 421(6923), 611-615 (2003).

2. S. Koke, C. Grebing, B. Manschwetus, and G. Steinmeyer, "Fast f-to-2f interferometer for a direct measurement of the carrier-envelope phase drift of ultrashort amplified laser pulses," Opt. Lett. 33(21), 2545-2547 (2008).

3. H. R. Telle, G. Steinmeyer, A. E. Dunlop, J. Stenger, D. H. Sutter, and U. Keller, "Carrier-envelope offset phase control: A novel concept for absolute optical frequency measurement and ultrashort pulse generation," Appl. Phys. B 69(4), 327-332 (1999).

4. M. Kakehata, H. Takada, Y. Kobayashi, K. Torizuka, Y. Fujihira, T. Homma, and H. Takahashi, "Single-shot measurement of carrier-envelope phase changes by spectral interferometry,” Opt. Lett. 26(18), 1436-1438 (2001).

5. A. Baltuska, M. Uiberacker, E. Goulielmakis, R. Kienberger, V. S. Yakovlev, T. Udem, T. W. Hansch, and F. Krausz, "Phase-controlled amplification of few-cycle laser pulses," IEEE J. Sel. Top. Quantum Electron. 9(4), 972-989 (2003).

6. C. Feng, J.-F. Hergott, P.-M. Paul, X. Chen, O. Tcherbakoff, M. Comte, O. Gobert, M. Reduzzi, F. Calegari, C. Manzoni, M. Nisoli, and G. Sansone, "Complete analog control of the carrier-envelope-phase of a high-power laser amplifier," Opt. Express 21(21), 25248-25256 (2013).

7. E. Cormier and P. Lambropoulos, “"'Effect of the initial phase of the field in ionization by ultrashort laser pulses," Eur. Phys. J. - At,” Mol. Opt. Plasma Phys. 2, 15-20 (1998).

8. T. Wittmann, B. Horvath, W. Helml, M. G. Schätzel, X. Gu, A. L. Cavalieri, G. G. Paulus, and R. Kienberger, "Single-shot carrier-envelope phase measurement of few-cycle laser pulses," Nat. Phys. 5(5), 357-362 (2009).

9. A. M. Sayler, T. Rathje, W. Müller, K. Rühle, R. Kienberger, and G. G. Paulus, "Precise, real-time, every-singleshot, carrier-envelope phase measurement of ultrashort laser pulses," Opt. Lett. 36(1), 1-3 (2011).

10. H. Wang, M. Chini, E. Moon, H. Mashiko, C. Li, and Z. Chang, "Coupling between energy and phase in hollowcore fiber based f-to-2f interferometers," Opt. Express 17(14), 12082-12089 (2009).

11. D. Hoff, F. J. Furch, T. Witting, K. Rühle, D. Adolph, A. M. Sayler, M. J. J. Vrakking, G. G. Paulus, and C. P. Schulz, "Continuous every-single-shot carrier-envelope phase measurement and control at $100 \mathrm{kHz}$," Opt. Lett. 43(16), 3850-3853 (2018).

12. P. V. Kelkar, F. Coppinger, A. S. Bhushan, and B. Jalali, "Time-domain optical sensing," Electron. Lett. 35(19), 1661-1662 (1999).

13. K. Goda and B. Jalali, "Dispersive Fourier transformation for fast continuous single-shot measurements," Nat. Photonics 7(2), 102-112 (2013). 
Optics EXPRESS

14. Y. C. Tong, L. Y. Chan, and H. K. Tsang, "Fibre dispersion or pulse spectrum measurement using a sampling oscilloscope," Electron. Lett. 33(11), 983-985 (1997).

15. P. Ryczkowski, M. Närhi, C. Billet, J.-M. Merolla, G. Genty, and J. M. Dudley, "Real-time full-field characterization of transient dissipative soliton dynamics in a mode-locked laser," Nat. Photonics 12(4), 221-227 (2018).

16. N. Thiré, R. Maksimenka, B. Kiss, C. Ferchaud, G. Gitzinger, T. Pinoteau, H. Jousselin, S. Jarosch, P. Bizouard, V. Di Pietro, E. Cormier, K. Osvay, and N. Forget, "Highly stable, $15 \mathrm{~W}$, few-cycle, 65 mrad CEP-noise mid-IR OPCPA for statistical physics," Opt. Express 26(21), 26907-26915 (2018).

17. P. Tournois, "Acousto-optic programmable dispersive filter for adaptive compensation of group delay time dispersion in laser systems," Opt. Commun. 140(4-6), 245-249 (1997).

18. F. Lücking, V. Crozatier, N. Forget, A. Assion, and F. Krausz, "Approaching the limits of carrier-envelope phase stability in a millijoule-class amplifier," Opt. Lett. 39(13), 3884-3887 (2014).

19. "R\&S®RTO2000 Oscilloscope User Manual," https://www.rohdeschwarz.com/webhelp/rto_html_usermanual_en/RTO_HTML_UserManual_en.htm.

20. J. Hult, R. S. Watt, and C. F. Kaminski, "Dispersion measurement in optical fibers using supercontinuum pulses," J. Lit. Technol. 25(3), 820-824 (2007).

21. N. I. Fisher and A. J. Lee, "A correlation coefficient for circular data," Biometrika 70(2), 327-332 (1983).

22. S. R. Jammalamadaka and A. Sengupta, Topics in Circular Statistics (World Scientific Pub Co Inc, 2001).

23. R. G. Lyons, Understanding Digital Signal Processing, II (Prentice Hall PTR, 2004). 